\title{
Nitrogen fixation and transportation by rhizobacteria: a scenario of rice and banana.
}

\begin{abstract}
Rhizobacteria can fix $\mathrm{N} 2$ in association with rice roots externally and internally and this fixed $\mathrm{N} 2$ is being utilized by the host plant. Nitrogen fixation and plant growth promotion by rhizobacteria might be important factors in achieving a sustainable rice production in the future. Attempt has been made to review recent research findings related to $\mathrm{N} 2$ fixation in rice by associative rhizobacteria and summarized thoroughly for a new research arena. The findings revealed that rhizobacteria are potential inocula for $\mathrm{N} 2$ fixation and utilization in rice and other non-legumineous like banana. The fixed N2 is converted to NH4+ ion in the cytoplasm of bacteria and excreted to the host cytoplasm through down hill process. This release can be inhibited by the presence of ambient NH4+. Most of the rhizospheric fixed N2 are being utilized after mineralization of bacterial dead body. Future research should not ignore the potential of improving rice production through endophytic rhizobial inoculation via mechanisms that involve Biological Nitrogen Fixation (BNF) process
\end{abstract}

Keyword: Assimilates; Colonization; N2-fixation; Quantification; Rhizobacteria; Rice; Trans location 